\title{
The Scope of Nutrition and Metabolism is Interacting More with Human Health
}

\author{
David Shiuan ${ }^{1,2,3 *}$ \\ ${ }^{1}$ Department of Nutrition and Metabolism, Bohai \\ University, College of Food Science and Engineering, \\ China \\ ${ }^{2}$ Department of Nutrition and Metabolism, Ningbo \\ University, Institute of Drug Discovery Technology, China \\ ${ }^{3}$ Department of Life Science, National Dong Hwa \\ University, China \\ *Corresponding author: David Shiuan, Department \\ of Nutrition and Metabolism, College of Food Science \\ and Engineering, Bohai University; Institute of Drug \\ Discovery Technology, Ningbo University, China; \\ Department of Life Science, National Dong Hwa \\ University, Hualien, Taiwan, China
}

Received: December 15, 2020; Accepted: J anuary 20 , 2021; Published: January 27, 2021

\section{Perspective}

During the past decades, we have witnessed a rapid rise in nutrition and metabolism-related disorders such as obesity and chronic diseases. Obesity has become a worldwide health issue characterized by increased consumption of animal fat and protein, refined grains, and added sugar, coupled with a more sedentary lifestyle. According to the 2017-2018 survey of the US National Center for Health Statistics (NCHS), the age-adjusted prevalence of obesity in adults was $42.4 \%$, with no significant differences between men and women or by age group. In order to battle the causes of chronic diseases, the promotion of healthy eating and active lifestyles has become an important topic within the scientific framework of nutrition and metabolism [1] Meanwhile, as worldwide human lifespan increased substantially in the past decades, aging has also become an important related issue. Now, knowledge from nutrition and metabolism has not only been used as the base to sustain human life, but also as critical scientific maneuvers to alleviate human diseases and to promote healthier lives. Several studies have indicated that Nicotinamide Adenine Dinucleotide (NAD) (Figure 1A) levels decrease with age and decline of NAD metabolism may induce several aging associated diseases, including metabolic and neurodegenerative diseases and various cancers [2]. It has been also discovered that compounds such as Sirtuins (SIRT) (Figure 1B), which are $\mathrm{NAD}^{+}$-dependent deacetylases, are involved in stress response, anti-oxidative defense, and longevity via posttranslational modifications [3]. The reduced sirtuin function and reduction of the cofactor $\mathrm{NAD}^{+}$were found to be closely associated with aging. On the other hand, resveratrol (Figure 1C), a natural polyphenolic compound widely found in grapes and wines, was found to display beneficial properties such as cardio-protective, antioxidant and anti-inflammatory activities [4]. These protective effects have been demonstrated through the activation of a NADdependent histone deacetylase family member Sirtuin-1 (SIRT-1) protein [5]. In addition, recent studies have shown that xanthohumol (Figure 1D) and its derivatives may improve obesity and metabolic syndrome in part by changing the gut microbiota and modulating bile acid metabolism [6]. Xanthohumol is an unique ingredient of hops (Humulus lupulus), also found in beer, which belongs to a class of compounds that contribute to the flavor of hops. Though being a beer-lover does not ensure weight loss or to healthy aging, a supplement may help. There is no doubt that a healthy lifestyle and good diet are the best means of sustaining life and alleviating various chronic diseases. The studies of the biochemical and genetic pathways that contribute to cellular damage accumulation over time have revealed many potential drug targets in dealing with aging and its related chronic diseases [7]. Currently, the discovery of the bioactive components from food ingredients can be accelerated by three major strategies: high throughput screening, phage display technique, and virtual screening techniques [8]. In silico target prediction and mass spectroscopy based proteomics, which are efficient strategies to identify and further validate the cellular targets, are also available. You may be aware that these powerful strategies still rely heavily upon the availability and completeness of libraries of potential drug candidates, especially the compounds from food ingredients. To achieve these very promising research goals, through the strategy of targeting the critical proteins involved in many chronic diseases, the establishment of an International Food Ingredients Consortium

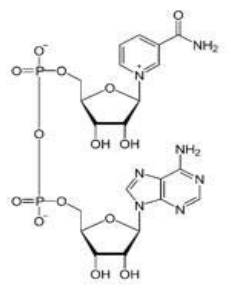

(A)

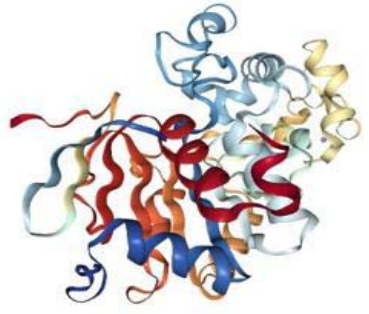

(B)

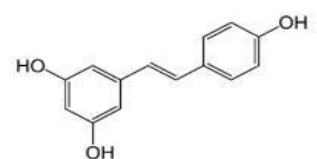

(C)

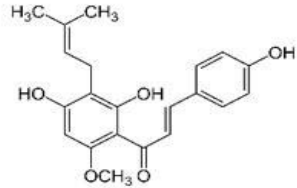

(D)

Figure 1: Molecular structures of (A) NAD+ (B) Sirtuin-1 (C) Resveratrol (D) Xanthohumol.

Austin J Nutr Metab - Volume 8 Issue 1 - 2021

Submit your Manuscript | www.austinpublishinggroup.com

Shiuan. () All rights are reserved
Citation: Shiuan D. The Scope of Nutrition and Metabolism is Interacting More with Human Health. Austin J Nutr Metab. 2021; 8(1): 1099. 
(IFIC) is thus proposed [8]. Taken together, now is the best time to discover the precious health-promoting ingredients hiding within food products. This endeavor may represent a great contribution for the ultimate goal of eating right and living a healthier life for people around the world.

\section{References}

1. Malik VS, Willett WC, Hu HB. Global obesity: trends, risk factors and policy implications. Nature Review in Endocrinology. 2013; 9: 13-27.

2. Braidy N, Berg J, Clement J, Khorshidi F, Poljak A, Jayasena T, et al. Role of nicotinamide adenine dinucleotide and related precursors as therapeutic targets for age-related degenerative diseases: rationale, biochemistry, pharmacokinetics, and outcomes. Antioxidants and Redox Signaling. 2019 30: 251-294

3. Kane AE, Sinclair DA. Sirtuins and $\mathrm{NAD}^{+}$in the development and treatment of metabolic and cardiovascular diseases. Circulation Research. 2018; 123 : 868-885.
4. Voloshyna I, Hussaini SM, Reiss AB. Resveratrol in cholesterol metabolism and atherosclerosis. Journal of Medicinal Food. 2012; 15: 763-773.

5. Morigi M, Perico L, Benigni A. Sirtuins in renal health and disease. Journal of American Society of Nephrology. 2018; 29: 1799-1809.

6. Zhang Y, Bobe G, Revel JS, Rodrigues RR, Sharpton TJ, Fantacone ML, et al. Improvements in metabolic syndrome by xanthohumol derivatives are linked to altered gut microbiota and bile acid metabolism. Molecular Nutrition \& Food Research. 2020; 64: e1900789.

7. Myersa A, Lithgow GJ. Drugs that target ageing: how do we discover them? Expert Opinion on Drug Discovery. 2019; 14: 541-548.

8. Shiuan D, Tai DF, Huang KJ, Yu ZP, Ni F, Li JR. Target-based discovery of therapeutic agents from food ingredients. Trends in Food Science \& Technology. 2020; 105: 378-384. 